\title{
Paediatric crossword puzzle - 20
}

Manouri P. Senanayake,

Sri Lanka Journal of Child Health, 2013; 42(3): 173

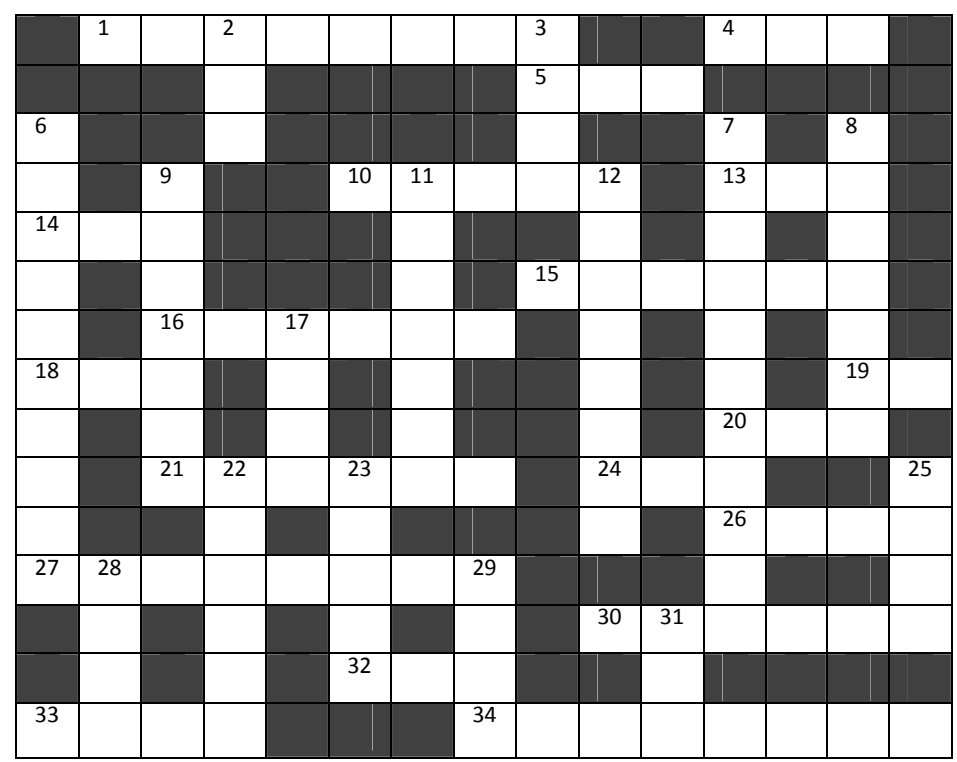

Across

1. endocrine disease postulated to be associated with vitamin D deficiency

4. agricultural chemical contaminating milk from New Zealand (abbrv.)

5. corrects SVT when applied to face

10. a measurement that suggests obesity

13. the main immunoglobulin in breast milk

14. a cause of prenatal hydronephrosis (abbrv.)

15. RNA virus that threatens to the world

16. most feared viral disease in Sri Lanka

18. virus transmitted by oro-oro route (abbrv.)

19. palliative shunt to increase pulmonary blood flow (abbrv.)

20. causes subcutaneous filariasis in humans

21. hypertonic solution that effectively reduces intracranial pressure

24. a test that detects antibodies (abbrv.)

26. retinal haemorrhage with white or pale centre

27. a post-op complication of ASD closure

30. poisonous nature of Russell's viper

32. chest compressions and rescue breathing

33. minimally invasive drainage of pleural cavity

(abbrv.)

34. poisonous predatory arthropod in Jaffna

\section{Down}

2. common leukaemia in Down syndrome (abbrv.)

3. infection with fever and leukocytosis (abbrv.)

6. a steroid sparing drug

7. dog filarial worm endemic in Sri Lanka

8. the most thalassemia prevalent province in Sri Lanka

9. multicentre randomized trial on treatment of nephrotic syndrome

11.binds prednisolone in blood stream

12.biomarker that detects myocardial damage

17. biomarker for early detection of acute renal failure

22. antifungals of low nephrotoxicity

23. the region of abdomen with signs of appendicitis

25. left over when milk coagulates

28. staphylococcal infections resistant to standard antibiotics (abbrv.)

29. respiratory illness spreading in Saudi Arabia

(Abbrv.)

31.a non-specific investigation (abbrv.)

Professor in Paediatrics, Faculty of Medicine, University of Colombo 